\title{
Measurement of the polarization profile across a surface-stabilized ferroelectric liquid crystal cell using the pyroelectric laser-intensity-modulation method
}

\author{
N. M. Shtykov \\ Department of Electronic and Electrical Engineering, Trinity College, University of Dublin, \\ Dublin 2, Ireland and Institute of Crystallography, Russian Academy of Sciences, 117333 Moscow, \\ Leninsky prosp. 59, Russia \\ J. K. Vij ${ }^{\mathrm{a})}$ \\ Department of Electronic and Electrical Engineering, Trinity College, University of Dublin, \\ Dublin 2, Ireland
}

(Received 28 May 2002; accepted 25 September 2002)

\begin{abstract}
The pyroelectric technique, called the laser-intensity-modulation method, was used for investigations of the spatial distribution of the pyroelectric coefficient that gives information about the director profile in ferroelectric liquid crystal (FLC) cells. The pyroelectric current from a cell, illuminated by the intensity-modulated laser beam, was measured as a function of the modulation frequency. The Tikhonov regularization procedure was used for the deconvolution of the pyroelectric current spectrum into the spatial profile of the pyroelectric coefficient. It was found that in the surface-stabilized ferroelectric liquid crystal (SSFLC) structure with a splayed director profile, the flexoelectric contribution to the local polarization has comparable magnitude with the spontaneous one. The effective flexoelectric coefficient of the investigated FLC material and the flexoelectric polarization of the SSFLC structure were determined. (c) 2003 American Institute of Physics. [DOI: 10.1063/1.1522746]
\end{abstract}

\section{INTRODUCTION}

There are two experimental methods that are most frequently used for measuring the spontaneous polarization of ferroelectric liquid crystal (FLC) materials. These are the reversal polarization current method using triangular ${ }^{1}$ or rectangular $^{2}$ voltage wave form, and the pyroelectric method, ${ }^{3,4}$ that include the continuous modulation ${ }^{5}$ and the impulse $^{6}$ techniques. These methods involve the application of a large voltage across the sample just sufficient to unwind a helical structure of the chiral smectic $\mathrm{C}\left(\mathrm{SmC}^{*}\right)$ phase and thus to create a uniform alignment of the spontaneous polarization in all of the smectic layers. In this uniform state there is no flexoelectric contribution ${ }^{7}$ to the local polarization of a chiral system in a ferroelectric $\mathrm{SmC}^{*}$ phase that is measured. Hence, the earlier mentioned techniques measure only a part of the averaged local polarization inherent in the $\mathrm{SmC}^{*}$ phase. The laser-intensity-modulation method (LIMM) pyroelectric technique, in spite of the complexity of its deconvolution methods, allows us at least in principle to investigate not only the spontaneous and the flexoelectric contributions to the total polarization of the ferroelectric $\mathrm{SmC}^{*}$ phase but also their spatial profiles of the different structures.

In this article, the LIMM pyroelectric technique, developed by Lang and Das-Gupta, ${ }^{8}$ was used for studying the spatial distribution of the local polarization and the molecular alignment in the ferroelectric SmC* phase. Recently this pyroelectric method was applied for studying the ferroelec-

a) Author to whom correspondence should be addressed; electronic mail: jvij@tcd.ie tric liquid-crystalline elastomers. ${ }^{9}$ The principle of the LIMM is as follows. ${ }^{8-11}$ An intensity-modulated laser beam irradiates an opaque electrode of a sample. Absorption of the laser light in the electrode produces thermal waves that extend into the sample which cause the pyroelectric response; this is a convolution of the pyroelectric coefficient distribution and the temperature profile across the sample. The penetration depth of the thermal waves depends on the modulation frequency and decreases with increase in the frequency. Higher is the modulation frequency, nearer to the heated electrode region of the sample is the pyroelectric response. Then, the measured pyroelectric current spectrum is deconvoluted to a spatial distribution of the pyroelectric coefficient. In mathematical sense, this task is the solution of the Fredholm's integral equation of the first kind, which belongs to the ill-posed problems. Deconvolution is carried out by using the Tikhonov regularization procedure, ${ }^{12}$ which has only recently been applied to the LIMM method. ${ }^{13}$

\section{EXPERIMENTAL TECHNIQUES}

The material used in our experiments was ferroelectric pitch-compensated mixture SCE13 (BDH). According to the specifications given by BDH (now E. Merck) this FLC mixture has the following phase transition sequence: isotropic $\left(100.8^{\circ} \mathrm{C}\right) \mathrm{N}^{*},\left(86.3^{\circ} \mathrm{C}\right) \mathrm{SmA}^{*}$, and $\left(60.8^{\circ} \mathrm{C}\right) \mathrm{SmC}^{*}$. The latter phase exists down to the room temperature.

A cell, used for the pyroelectric measurements, consisted of two glass plates with indium tin oxide (ITO) layers as electrodes and the Mylar thin-film stripes of $15 \mu \mathrm{m}$ thickness as spacers (Fig. 1). One of the ITO layers was coated with 


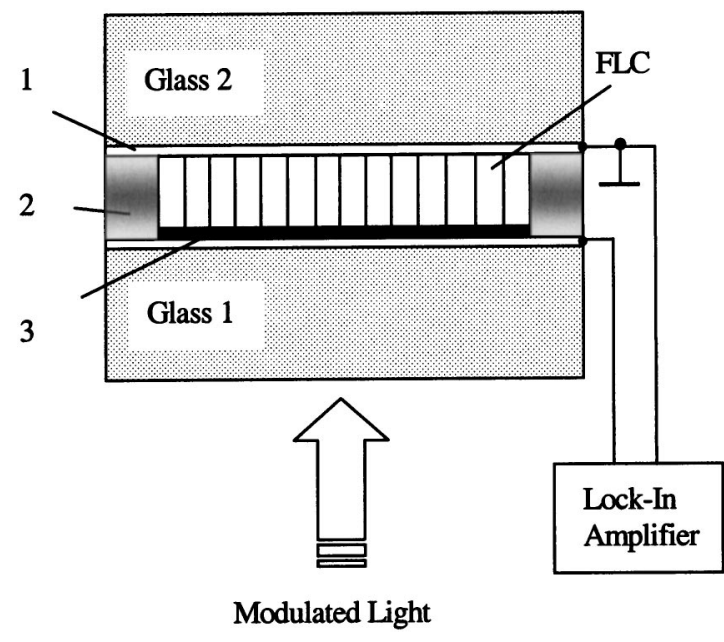

FIG. 1. Schematic of the liquid crystal cell and the experimental setup. 1-ITO, 2-Mylar film, 3-ITO+ Al film. The pyroelectric current is measured by a lock-in amplifier SR830 (Stanford Research Systems) with its internal current-to-voltage converter

50-nm-thick aluminum film using vacuum evaporation. A set of rectangular electrodes of dimensions $4 \times 20 \mathrm{~mm}^{2}$ was arranged cross-like in the cell. Hence, the working area of electrodes was $4 \times 4 \mathrm{~mm}^{2}$. For homogeneous alignment of the liquid crystalline sample, the conducting surfaces of the glass plates were spin coated with polyimide film (RN-1266, Nissan Chemical Industry), these were cured for a duration of $1 \mathrm{~h}$ at a temperature of $250{ }^{\circ} \mathrm{C}$ and then both rubbed in one direction. The cell was heated and filled with the FLC mixture in the isotropic phase and cooled slowly to the ferroelectric $\mathrm{SmC}^{*}$ phase.

Figure 1 shows a schematic diagram of the pyroelectric cell used in the pyroelectric measurements. The pyroelectric current is fed to the lock-in-amplifier as before. ${ }^{14}$ The sample is heated with an amplitude modulated light beam generated by a semiconductor diode laser module. The amplitude modulated light beam modulated a frequency of $f$ is absorbed by the opaque electrode (aluminum and ITO films) on the upper side of the lower glass plate and causes an oscillation in temperature of the electrode and its surroundings, i.e., liquid crystal and glass plates. The oscillating temperature produces alternating pyroelectric current in a sample. A diode laser module with the wavelength of $685 \mathrm{~nm}$ and the $\mathrm{cw}$ output power of $19.5 \mathrm{~mW}$ was used as a modulated light source. The current through the laser diode module is regulated by TTL logic, consequently the intensity of its light beam follows approximately to that of a rectangular wave form. Lock-in amplifier with internal current-to-voltage converter measures the rms magnitudes of the real and the imaginary components of the pyroelectric current at the first harmonic of the modulation signal.

\section{THEORETICAL BACKGROUND}

\section{A. Model of temperature distribution within the sample}

The coordinate system is defined such that the axis $x$ is along the cell thickness, $z$ axis is along the smectic layer normal, the $y$ axis is along the glass plate; $x, y$ is the smectic layer plane and $y, z$ is the plane of the glass plate. This will be shown on the diagram later. The heating by laser of the opaque electrode $(x=0)$ produces a heat flux $q\left(\right.$ in $\left.\mathrm{W} / \mathrm{cm}^{2}\right)$ given at the first harmonic of the modulating TTL voltage by the expression $q(0, t)=q_{0}[1+\cos (\omega t)]$, where $q_{0}$ $=4 I_{0} \eta / \pi, I_{0}\left(i n \mathrm{~W} / \mathrm{cm}^{2}\right)$ is the $\mathrm{cw}$ intensity of laser beam, $\omega$ is the radial frequency, and $\eta$ is the coefficient of the absorption of light in the electrode. The heat flux consists of a constant term plus a periodic one. Since the diameter of the laser beam is assumed to be much greater than the sample thickness, the one-dimensional heat-conduction equation is applicable to the problem under discussion. Temperature increment $\delta T$, produced by the laser heating can be expressed as the sum of a term $T_{c}$ due to the constant component of the heat flux and a term $T_{m}$ caused by the modulated component of the heat flux. It is known ${ }^{8}$ that $T_{c}$ distribution decays to a steady spatial distribution with exponential time dependence for a time of approximately $1 \mathrm{~ms}$ and therefore cannot contribute to the sinusoidal response measured in our experiment.

We have examined an applicability of several thermal models to our case, some of these are described in the literature, ${ }^{8,9,11}$ and these were applied to the geometry of the liquid crystal cell. It was found, however, that the model given by Leister et al. ${ }^{9}$ gives the most reliable results for the deconvolution of the experimental data using the regularization procedure

$$
\begin{aligned}
& T_{m}(x, t)=q_{0} \frac{k \sigma \cosh (\sigma(d-x))+H_{d} \sinh [\sigma(d-x)]}{\left[(k \sigma)^{2}+H_{0} H_{d}\right] \sinh (\sigma d)+k \sigma\left(H_{0}+H_{d}\right) \cosh (\sigma d)} e^{i \omega t}, \\
& \sigma=(1+i) \sqrt{\omega / 2 D} .
\end{aligned}
$$

Here $T_{m}(x)$ is the profile of the modulated temperature in the sample and $d$ is the thickness of the liquid crystal sample. $D$ and $k$ are the thermal diffusivity and the conductivity of the liquid crystal. The thermal diffusivity $D=k / c \rho$ is expressed through thermal conductivity $k$, specific heat per mass $c$, and density $\rho$ of a material. $H_{0}$ and $H_{d}$ are the heat transfer coefficients on the front and the rear boundary of the sample and $i$ is the imaginary operator. 


\section{B. Pyroelectric current}

The pyroelectric current is given by the expression ${ }^{8,10}$

$$
J_{m}(t)=J_{m 0}(f) e^{i \omega t}=\frac{A}{d} \int_{0}^{d} p(x) \frac{\partial T_{m}(x, t)}{\partial t} d x,
$$

where $A$ is the electrode area and $p(x)$ is a spatially dependent pyroelectric coefficient. This coefficient describes the rate of change $\Delta Q / \Delta T$ of the surface charge $\Delta Q$ produced by a temperature change $\Delta T$. The various terms that contribute to the pyroelectric coefficient are given by ${ }^{15}$

$$
p(x)=\alpha_{P} P(x)-\left(\alpha_{x}-\alpha_{\varepsilon}\right) \varepsilon_{0} \varepsilon E(x),
$$

where $\varepsilon_{0}$ is the permittivity of vacuum, $\varepsilon$ is the relative permittivity of the investigated material, and $E(x)$ is the electric field. The coefficients $\alpha_{P}, \alpha_{x}$, and $\alpha_{\varepsilon}$ denote the relative temperature dependencies of the polarization, the thermal expansion, and the permittivity of the sample, respectively. A spatial dependence of the pyroelectric coefficient may be caused by a spatial dependence of the spontaneous polarization or by free charges, intrinsic or injected from the electrodes. ${ }^{8}$ Only in the case when the polarization charge $\operatorname{div} \boldsymbol{P}$ is locally compensated by a free charge density $\rho(x)$, i.e., $\operatorname{div} \boldsymbol{P}=\rho$, Eq. (3) reduces ${ }^{16}$ to the well-known expression $p(x)=\alpha_{P} P(x)=\partial P / \partial T$. We believe that in most liquid-crystalline materials having a free charge concentration of $\sim 10^{15} \mathrm{~cm}^{-3}$, this is indeed the case, at least in the absence of an external electric field.

Inserting the temperature distribution from Eq. (1) into Eq. (2) we obtain

$$
J_{m 0}(f)=\frac{i \omega A q_{0}}{d} \int_{0}^{d} p(x) \frac{k \sigma \cosh (\sigma(d-x))+H_{d} \sinh (\sigma(d-x))}{\left[(k \sigma)^{2}+H_{0} H_{d}\right] \sinh (\sigma d)+k \sigma\left(H_{0}+H_{d}\right) \cosh (\sigma d)} d x,
$$

where $J_{m 0}(f)$ is frequency dependent complex amplitude of the pyroelectric current.

\section{Regularization technique}

In a LIMM experiment, the pyroelectric current $J_{m 0}(f)$ is measured as a function of the modulation frequency $f$. The goal of these measurements is to retrieve the pyroelectric coefficient $p(x)$ contained in the integral on the right-hand side of Eq. (4), which is a Fredholm integral equation of the first kind. The deconvolution of this type of equation is generally viewed as an ill-posed mathematical problem. One of the best methods, providing stable and reliable solution of this type of problem, is the Tikhonov regularization method. ${ }^{12}$ It implies that to find the real pyroelectric coefficient distribution $p(x)$ we should optimize the following expression:

$$
\Psi(\alpha)=\sum_{n=1}^{N_{f}}\left[J\left(f_{n}\right)-J_{m 0}\left(f_{n}, p_{\alpha}\right)\right]^{2}+\alpha\left\|L p_{\alpha}\right\|,
$$

where $J\left(f_{n}\right)$ are the experimental data, $J_{m 0}\left(f_{n}, p_{\alpha}\right)$ are the data calculated using Eq. (4). These are the calculated magnitudes of the pyroelectric current at frequencies $f_{n}$ and for pyroelectric distribution $p_{\alpha}(x) . \alpha$ and $L$ are the regularization parameter and the operator, respectively. $p_{\alpha}(x)$ is an approximation of the real distribution $p(x)$ for a regularization parameter $\alpha$ and $N_{f}$ is a number of experimental points (which are the number of frequencies). We used the first derivative of the function $p_{\alpha}(x)$ in the regularization term $L p_{\alpha}(x)$ for getting of smooth spatial distribution. The norm $\left\|L p_{\alpha}\right\|$ defined in the earlier equation is given as

$$
\left\|L p_{\alpha}\right\|=\int_{0}^{d}\left(\frac{d p_{\alpha}}{d x}\right)^{2} d x .
$$

The first term in Eq. (5) minimizes the deviations between the experimental data and those predicted theoretically, whereas the second term affects the smoothness of the curve that approximates $p(x)$. The choice of the regularization parameter $\alpha$ is very important for the quality and the reliability of the $p_{\alpha}(x)$ fit. Several principal methods are involved in the choice of the regularization parameter. ${ }^{12,17}$ The principle of the Honerkamp-Weese self-consistency method ${ }^{17}$ is based on the assumption that the optimal regularization parameter $\nu$ gives the minimum value of the norm $D(\alpha)=\left\|p_{\nu}-p_{\alpha}\right\|$. We use a simpler method based on their basic concept and state that the regularization parameter is optimal if it gives the minimum norm $\left\|d p_{\alpha} / d \alpha\right\|$.

To find the applicability of this method to our problem, we have selected one of the typical distributions occurring in chiral ferroelectric liquid crystals $p(x)=p_{0} \cos \left(\varphi_{0}+\pi x / d\right)$, where $p_{0}=1 \mathrm{nC} / \mathrm{cm}^{2} \mathrm{~K}$ and $\varphi_{0}=-100^{\circ}$. Using this pyroelectric distribution and Eq. (4) the simulated currentfrequency spectra were calculated. These are shown in Fig. 2(a). Then polarization distribution was calculated from the real $J_{\mathrm{Re}}$ and the imaginary $J_{\mathrm{Im}}$ parts of the pyroelectric current using the regularization method described earlier [Eqs. (5) and (6)]. The initial distribution of the pyroelectric coefficient (solid line) and the pyroelectric coefficient profiles calculated using the regularization procedure, are shown in Fig. 2(b). We note that the results so obtained fit the initial distribution rather well except in small regions close to the front and the rear surfaces of a cell. These prove the validity and the reliability of our numerical procedure and more specifically provide the correctness of the regularization parameter choice method used here.

\section{Local polarization in FLC material}

Molecules in a smectic layer of a FLC material are tilted with respect to the layer normal [Fig. 3(a)]. The coordinate 

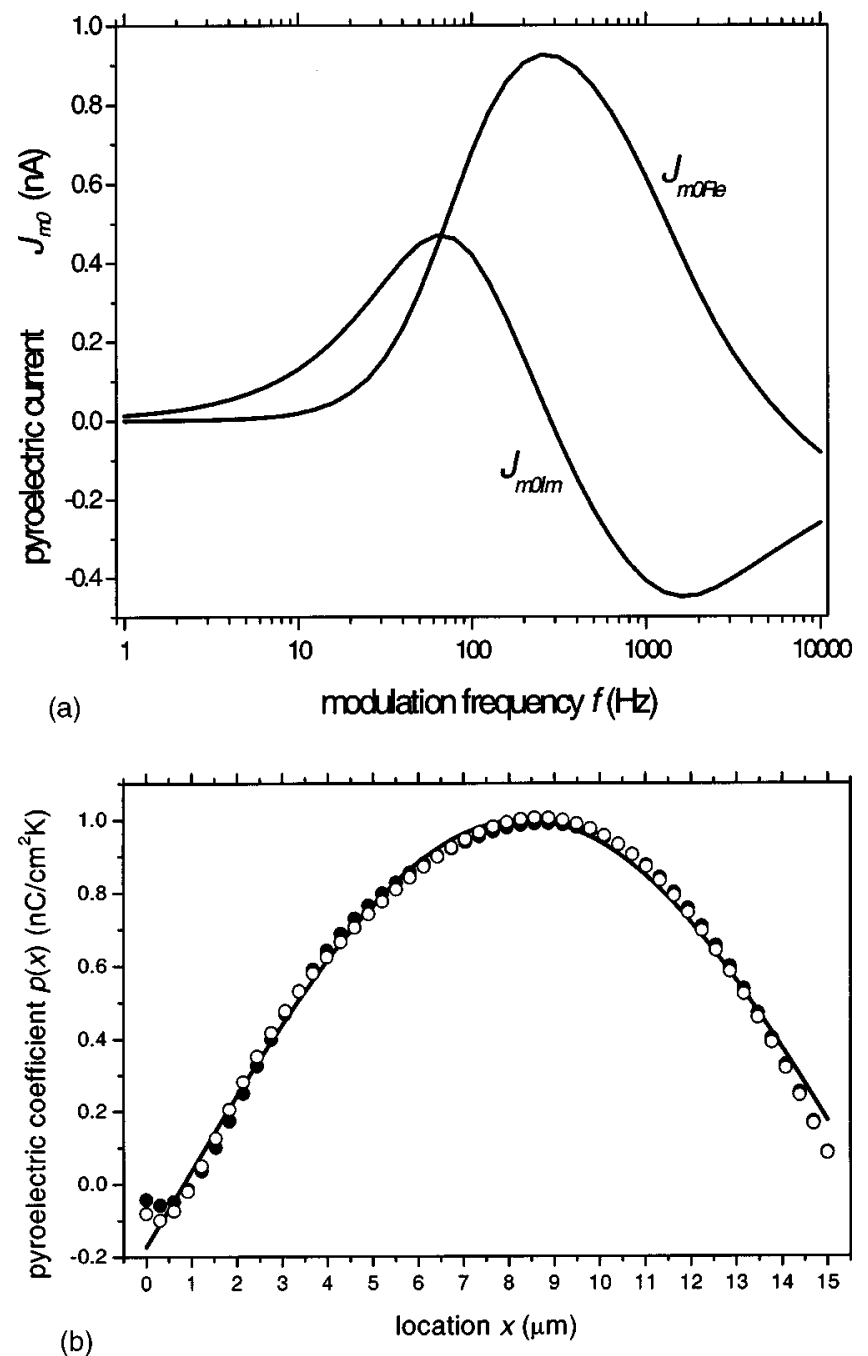

FIG. 2. (a) The frequency spectra of the real $J_{m 0 \text { Re }}$ and the imaginary $J_{m 0 \mathrm{Im}}$ parts of the pyroelectric current calculated for the pyroelectric coefficient distribution of $p(x)=p_{0} \cos \left(\varphi_{0}+\pi x / d\right)$, where $p_{0}=1 \mathrm{nC} / \mathrm{cm}^{2} \mathrm{~K}$ and $\varphi_{0}=$ $-100^{\circ}$. The parameters used for the calculation were: $I_{0}=2800 \mathrm{~W} / \mathrm{m}^{2}, \eta$ $=0.43$, sample thickness $d=15 \mu \mathrm{m}$, thermal conductivity $k$ $=0.12 \mathrm{~W} / \mathrm{m} \mathrm{K}$, and diffusivity $D=5.3 \times 10^{-8} \mathrm{~m}^{2} / \mathrm{s}$ of a liquid crystal (see Ref. 21) and the heat transfer coefficients are $H_{0}=1.1 \mathrm{~W} / \mathrm{m}^{2} \mathrm{~K}$ and $H_{d}$ $=1.0 \mathrm{~W} / \mathrm{m}^{2} \mathrm{~K}$. (b) The pyroelectric profiles obtained from the real (solid circles) and the imaginary (open circles) parts of the simulated pyroelectric current spectrum by using the regularization procedure and the initial pyroelectric coefficient distribution (solid line).

system of the axes is also shown in this figure. The vector $\boldsymbol{n}$ denoting the director indicates the tilt direction and is described by the polar tilt angle $\theta$ and the azimuthal angle $\varphi$. Local polarization in FLC materials in the absence of external electric field consists of two parts. The first part is the spontaneous polarization $P_{S}$ whose magnitude is coupled with the tilt angle $\theta$. The direction of the vector $\mathbf{P}_{S}$ is always perpendicular to the tilt plane and is given by the expression $\mathbf{P}_{S}=P_{0}(\mathbf{z} \cdot \mathbf{n})[\mathbf{z} \times \mathbf{n}]$, where $\mathbf{z}$ is the layer normal and $P_{0}$ is a material parameter. ${ }^{18}$ If vectors $\mathbf{P}_{S}, \mathbf{z}$ and $\mathbf{n}$ make a righthanded system then the FLC material is defined to have positive $\mathbf{P}_{S}$ and the parameter $P_{0}>0$. The second part of the local polarization is the flexoelectric polarization $P_{F}$, which may approximately be of the same order of magnitude as $P_{S}$. The flexoelectric polarization appears in liquid crystals for the

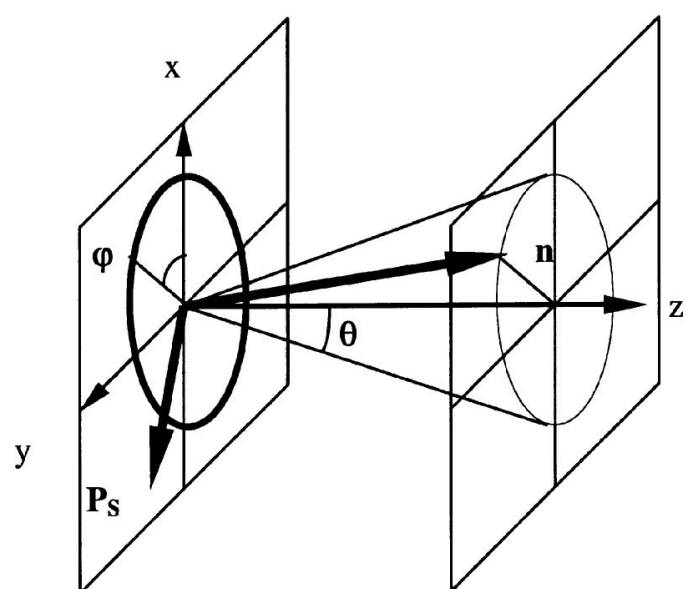

(a)
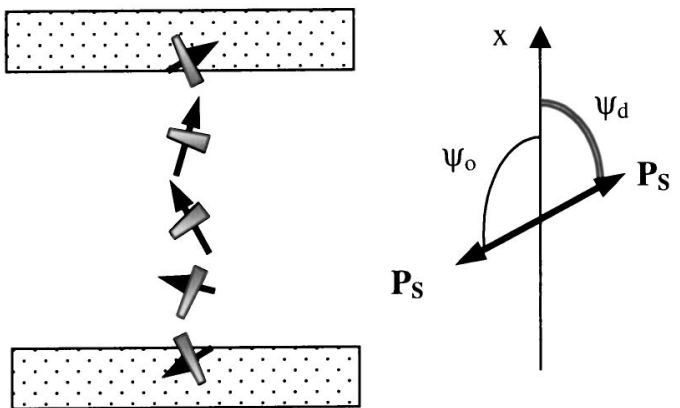

(b)

FIG. 3. (a) Definitions of the azimuthal $\varphi$ and the tilt $\theta$ angles. These describe the orientation of the director $\mathbf{n}$ and the spontaneous polarization vector $\mathbf{P}_{s}$ in the smectic layer. Axes are defined, $x-y$ plane is parallel to the smectic layers, $z$ is along the layer normal. (b) The spontaneous polarization distribution in a splayed SSFLC structure in the smectic layer $x-y$. The azimuthal angles of the polarization at the bottom $(x=0)$ and at the top $(x=d)$ boundaries are $\psi_{0}$ and $\psi_{d}=\psi_{0}+180^{\circ}$, respectively. The layer normal, i.e., $z$ direction, is perpendicular to the plane of the paper.

cases when the field of the director $\mathbf{n}(x, y, z)$ is not uniform ${ }^{19}$ and is given by the expression $\mathbf{P}_{F}=e_{s} \mathbf{n}(\nabla \cdot \mathbf{n})+e_{b} \overrightarrow{\mathbf{n}} \times[\nabla$ $\times \overrightarrow{\mathbf{n}}]$, where $e_{s}$ and $e_{b}$ are the flexoelectric coefficients for the splay and the bend deformations, respectively.

It is well known that surface interactions in thin-layer FLC cells unwind the helical structure of the SmC* phase $^{20}$ and produce the so called surface-stabilized ferroelectric liquid crystal (SSFLC) structures. In very thin samples, the SSFLC structure is characterized by the uniform director distribution and a uniform spontaneous polarization profile. In more thick samples the nonuniform or the so-called "splayed structures" are more stable. In a splayed structure, the azimuthal angle of the spontaneous polarization $\psi$ changes across the sample from its value at the bottom $\psi_{0}$ to the top boundaries $\psi_{d}$ [Fig. 3(b)] of the cell. The azimuthal angles of the polarization and the director are connected to each other through the equation $\psi=\varphi \pm \pi / 2$ [Fig. 3(a)], where the signs "+ and -" correspond to the FLCs with positive and negative spontaneous polarizations.

The pitch-compensated FLC mixture investigated in our work produces the splayed SSFLC structure in the sample of $15 \mu \mathrm{m}$ thickness. In the geometry of our experiment (Figs. 1 
and 3) the components of the spontaneous polarization $P_{S x}$ and the flexoelectric polarization $P_{F x}$ along the $x$ axis are given as $P_{S x}=-\left(P_{0} / 2\right) \sin 2 \theta \sin \varphi$ and $P_{F x}=1 / 2\left(e_{b}\right.$ $\left.-e_{s}\right) \sin ^{2} \theta \sin 2 \varphi(d \varphi / d x)$. Therefore, the projection of the total local polarization on the $x$ axis is expressed as

$$
\begin{aligned}
P_{x}(x)= & -\frac{P_{0}}{2} \sin 2 \theta \sin \varphi(x) \\
& +\left(\frac{e_{b}-e_{s}}{2}\right) \sin ^{2} \theta \sin 2 \varphi(x) \frac{d \varphi(x)}{d x} .
\end{aligned}
$$

In principle, in this expression there are three temperature dependent parameters. These are the two flexoelectric coefficients $e_{s}$ and $e_{b}$, and the tilt angle $\theta$. The latter has a strong temperature dependence, which is given approximately by $\left(T_{A C}-T\right)^{\beta}$. Here $T_{A C}$ is a temperature of the phase transition from the paraelectric $\mathrm{SmA}^{*}$ to the ferroelectric $\mathrm{SmC}^{*}$ phase and the exponent $\beta$ usually varies from 0.3 to 0.5 . Due to the absence of the information regarding the temperature dependence of the flexoelectric coefficients in $\mathrm{SmC}^{*}$ phase, we will consider these to be independent of temperature in what follows. In this case, the pyroelectric coefficient $p(x)$ obtained from the Eq. (7) can be expressed as

$$
\begin{aligned}
p(x)= & \frac{d \theta}{d T}\left[-P_{0} \cos 2 \theta \sin \varphi(x)\right. \\
& \left.+\left(\frac{e_{b}-e_{s}}{2}\right) \sin 2 \theta \sin 2 \varphi(x) \frac{d \varphi(x)}{d x}\right] .
\end{aligned}
$$

This equation will be used for fitting the experimentally obtained distribution of the pyroelectric coefficient across the sample.

\section{EXPERIMENTAL RESULTS AND DISCUSSION}

Experimental measurements were carried out in the $\mathrm{SmC}^{*}$ phase of a FLC mixture SCE13 at room temperature $\left(24^{\circ} \mathrm{C}\right)$. Pyroelectric current has been measured in the frequency range from $1 \mathrm{~Hz}$ to $5 \mathrm{kHz}$ at ten points per decade of the frequency spectrum. Figure 4(a) shows the frequency spectra for the real $J_{\mathrm{Re}}$ and the imaginary $J_{\text {Im }}$ parts of the pyroelectric current. The real part of the pyroelectric spectrum shows a maximum at a frequency of $\sim 200 \mathrm{~Hz}$ and then drops down to a lower value. This result allows one to state qualitatively that the pyroelectric coefficient near the front surface of the cell is lower than in the middle of the cell and consequently the polarization profile of the investigated SSFLC structure is not uniform. The profiles of the pyroelectric coefficient $p(x)$ calculated from the real and the imaginary parts of the pyroelectric current using the regularization procedure are given in Fig. 4(b). The spectra of the pyroelectric current $J_{m 0}$ recalculated for these profiles using Eq. (4) are presented in Fig. 4(a) by the solid lines. The norm of the deviation between the measured and the calculated spectrum is about $2.5 \%$ for the real and the imaginary parts of the current. These $p(x)$ profiles monotonously decrease when reaching the two boundaries. Near the front boundary the profiles obtained for real and imaginary components of the pyroelectric current have a maximal deviation.
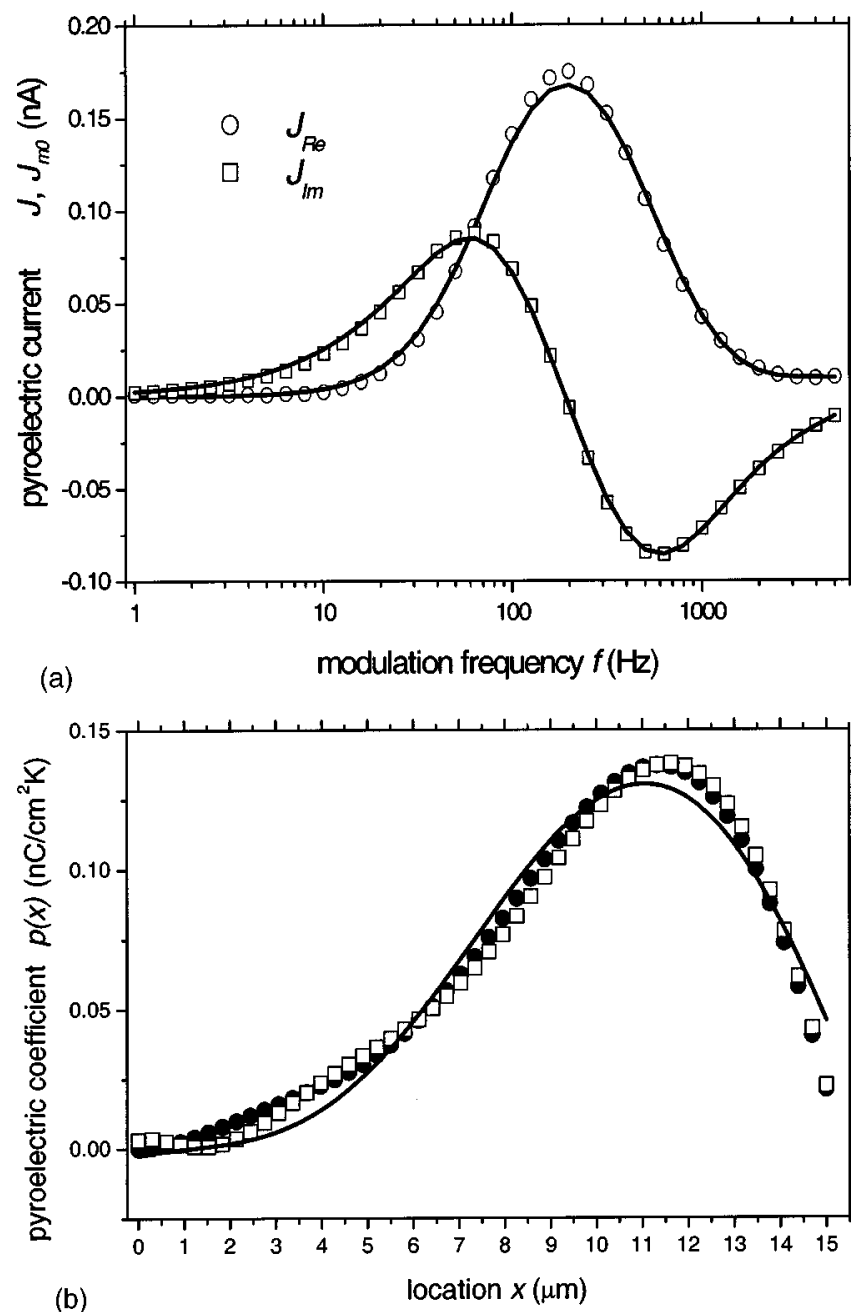

FIG. 4. (a) The real $J_{\mathrm{Re}}$ and the imaginary $J_{\mathrm{Im}}$ parts of the pyroelectric current spectra measured for a SSFLC sample and those $\left(J_{m 0 \text { Re }}\right.$ and $\left.J_{m 0 \mathrm{Im}}\right)$ recalculated by using Eq. (4) for the determined pyroelectric coefficient profiles. (b) The pyroelectric coefficient distributions calculated from the real (solid circles) and the imaginary (open squares) parts of the pyroelectric current spectra using the regularization procedure and the best-fit curve obtained with the aid of Eq. (8).

A priori it is apparent from the obtained results that the director distribution in the SSFLC sample is not uniform. For the uniform director distribution, the pyroelectric profile would have been found to be flat across the cell thickness with a nonzero amplitude. Therefore, the director has different orientations at the boundaries of the FLC cell, these are characterized by the azimuthal angles $\varphi_{0}$ and $\varphi_{d}$ on the front and the rear boundaries of the cell, respectively. In the absence of the external electric field the transition from the orientation at the front boundary to the rear goes through a uniform rotation in the azimuthal angle, with a constant rate of $d \varphi / d x=\left(\varphi_{d}-\varphi_{0}\right) / d$. In the case of the symmetrical boundary conditions $\varphi_{d}-\varphi_{0}= \pm 180^{\circ}$. Further we will consider this case. To find out the details of the director distribution and the contribution ratio of the spontaneous and the flexoelectric polarization to the total polarization in the investigated SSFLC sample, we fitted the experimental data using Eq. (8). The fitting function was taken in the form of $p(x)=B_{s} \sin \varphi(x)+B_{f} \sin 2 \varphi(x)$ with $\varphi(x)=\varphi_{0}+x \cdot \pi / d$. The 
fitting parameters were $B_{s}, B_{f}, \varphi_{0}$. The parameters $B_{s}, B_{f}$ describe the contributions of the spontaneous and the flexoelectric polarizations, respectively, whereas $\varphi_{0}$ is the azimuthal angle of the director on the front boundary of the cell. The best-fit parameters are found to be $B_{s}$ $=0.103 \mathrm{nC} / \mathrm{cm}^{2} \mathrm{~K}, \quad B_{f}=-0.048 \mathrm{nC} / \mathrm{cm}^{2} \mathrm{~K}, \quad$ and $\varphi_{0}=$ $-13.5^{\circ}$. The corresponding curve is presented in Fig. 4(b) by a solid line. These results show that the contribution of the flexoelectric polarization to the total pyroelectric coefficient is approximately two times lower than that for the spontaneous one.

From the data sheet of the SCE13 mixture given by the manufacturer, the value of the tilt angle and its temperature derivative $d \theta / d T$ were estimated to be equal to $21.6^{\circ}$ and $-0.0018 \mathrm{rad} / \mathrm{K}$, respectively. Using these parameters and the best-fit values of the parameters $B_{s}$ and $B_{f}$, the coefficient of the spontaneous polarization $P_{0}=81 \mathrm{nC} / \mathrm{cm}^{2}$ and the effective flexoelectric coefficient $\left(e_{b}-e_{s}\right)=-0.038 \mathrm{nC} / \mathrm{cm}=3.8$ $\times 10^{-11} \mathrm{C} / \mathrm{cm}$ were calculated. The modulus of the spontaneous polarization $P_{s}=\left(P_{0} / 2\right) \sin 2 \theta$ calculated with the obtained parameter $P_{0}$ is found to be $27.5 \mathrm{nC} / \mathrm{cm}^{2}$, which agrees perfectly with the value specified by the manufacturer for the SCE13 mixture $\left(27.8 \mathrm{nC} / \mathrm{cm}^{2}\right.$ at $\left.20^{\circ} \mathrm{C}\right)$. The magnitude of the flexoelectric polarization $P_{F}=1 / 2\left(e_{b}\right.$ $\left.-e_{s}\right) \sin ^{2} \theta(d \varphi / d x)$ is $5.3 \mathrm{nC} / \mathrm{cm}^{2}$, which approximately is $20 \%$ of the spontaneous polarization. In very thin samples $(1.5 \mu \mathrm{m})$ that are used for the electro-optical studies, for example in the investigations of the cells that exhibit $V$ shaped switching, this contribution should be about ten times higher due to a large value of $d \varphi / d x$.

In conclusion, the LIMM pyroelectric technique with Tikhinov regularization procedure was used for the determination of the spatial distribution of the director in the SSFLC cell. It was found that the flexoelectric polarization makes an important contribution to the local polarization and cannot be neglected. The magnitude of the flexoelectric polarization was determined for the investigated FLC material, and was found to be about $20 \%$ of the spontaneous polarization.

\section{ACKNOWLEDGMENT}

This work was supported by the Enterprise Ireland Basic Research Grant No. SC/2000/302.

${ }^{1}$ K. Miyasato, S. Abe, H. Takezoe, A. Fukuda, and E. Kuze, Jpn. J. Appl. Phys. 22, L661 (1983).

${ }^{2}$ V. M. Vaksman and Yu. P. Panarin, Mol. Mater. 1, 147 (1992).

${ }^{3}$ A. G. Chynoweth, J. Appl. Phys. 27, 78 (1956).

${ }^{4}$ L. M. Blinov, in Advances in Liquid Crystals: A Special Volume of Advances in Chemical Physics, edited by J. K. Vij (Wiley, New York, 2000), Vol. 113, pp. 77-158.

${ }^{5}$ A. M. Glass, J. S. Patel, J. W. Goodby, D. H. Olson, and J. M. Geary, J. Appl. Phys. 60, 2778 (1986).

${ }^{6}$ L. M. Blinov, L. A. Beresnev, N. M. Shtykov, and Z. M. Elashvili, J. Phys. (France) 40, C3 (1976).

${ }^{7}$ S. T. Lagerwall, Ferroelectric and Antiferroelectric Liquid Crystals (Wiley, Weinheim, 1999), p. 67.

${ }^{8}$ S. B. Lang and D. K. Das-Gupta, J. Appl. Phys. 59, 2151 (1986).

${ }^{9}$ N. Leister, W. Lehmann, U. Weber, D. Geschke, F. Kremer, P. Stein, and H. Finkelmann, Liq. Cryst. 27, 289 (2000).

${ }^{10}$ S. Bauer and B. Ploss, J. Appl. Phys. 68, 6361 (1990).

${ }^{11}$ S. Bauer, J. Appl. Phys. 75, 5306 (1994).

${ }^{12}$ A. N. Tikhonov and V. Y. Arsenin, Solution of Ill-Posed Problems (Wiley, New York, 1977).

${ }^{13}$ P. Bloß, M. Steffen, H. Schäfer, G.-M. Yang, and G. M. Sessler, J. Phys. D 30, 1668 (1997).

${ }^{14}$ A. Kocot, R. Wrzalik, J. K. Vij, and R. Zentel, J. Appl. Phys. 75, 728 (1994).

${ }^{15}$ R. Gerhard-Multhaupt, Ferroelectrics 75, 385 (1987).

${ }^{16}$ B. Ploss, R. Emmerich, and S. Bauer, J. Appl. Phys. 72, 5363 (1992).

${ }^{17}$ J. Honerkamp and J. Weese, Continuum Mech. Theomodyn. 2, 17 (1990).

${ }^{18}$ S. A. Pikin, Strucural Transformations in Liquid Crystals (Gordon and Breach, New York, 1991), Chap. V.

${ }^{19}$ R. B. Meyer, Phys. Rev. Lett. 22, 918 (1969).

${ }^{20}$ N. A. Clark, M. A. Handschy, and S. T. Lagerwall, Mol. Cryst. Liq. Cryst. 94, 213 (1983).

${ }^{21}$ M. Marinelli, F. Mercuri, S. Foglietta, U. Zammit, and F. Scudieri, Phys. Rev. E 54, 1604 (1996). 